\title{
Cortical fMRI activation to opponents' body kinematics in sport-related anticipation: expert-novice differences with normal and point-light video
}

\author{
M. J. Wright ${ }^{1, \mathrm{a}}$ D. T. Bishop ${ }^{1,2}$ R. C. Jackson ${ }^{2,3}$ B. Abernethy $y^{3,4}$ \\ ${ }^{1}$ Centre for Cognition and Neuroimaging, Brunel University, Uxbridge, Middlesex, UK, ${ }^{2}$ Centre \\ for Sports Medicine and Human Performance, Brunel University, Uxbridge, Middlesex, UK, \\ ${ }^{3}$ Institute of Human Performance, Hong Kong University, Pokfulam, Hong Kong, ${ }^{4}$ School of \\ Human Movement Studies, University of Queensland, St. Lucia, Australia. \\ ${ }^{a}$ Corresponding author: Michael J. Wright, PhD., Centre for Cognition and Neuroimaging, \\ Department of Psychology, Brunel University, Kingston Lane, Uxbridge, Middlesex, UB8 3PH, \\ UK.E-mail: michael.wright@brunel.ac.uk.Phone: +44 1895265957
}

Keywords: fMRI, sport, expertise, biological motion, anticipation, mirror neuron system.

\begin{abstract}
Badminton players of varying skill levels viewed normal and point-light video clips of opponents striking the shuttle towards the viewer; their task was to predict in which quadrant of the court the shuttle would land. In a whole-brain fMRI analysis we identified bilateral cortical networks sensitive to the anticipation task relative to control stimuli. This network is more extensive and localised than previously reported. Voxel clusters responding more strongly in experts than novices were associated with all task-sensitive areas, whereas voxels responding more strongly in novices were found outside these areas. Task-sensitive areas for normal and point-light video were very similar, whereas early visual areas responded differentially, indicating the primacy of kinematic information for sport-related anticipation.
\end{abstract}

\section{Introduction}

In sports such as tennis and badminton, there is insufficient time to fully analyse the trajectory of the projectile before making a preparatory response for a return shot. Consequently there is a strong premium on anticipation based on the opponent's bodily movements; perceptual expertise entails the early identification of such cues. Experiments in which a video of an opponent's action is curtailed at various times relative to ball-racket contact (temporal occlusion) have demonstrated the importance of the opponent's body kinematics for predicting the direction and forcefulness of the shot [1-5].

Performance in temporal occlusion tasks correlates strongly with expertise in the relevant sport. An important issue is to establish what information is used for the anticipation task. Behavioural experiments using point-light displays (dots of light depicting the motion of key joint centres and equipment) $[2,4,5]$ demonstrate that observers can use the purely kinematic information of the player's body movement. It was found $[4,5]$ that despite some loss of accuracy, the time course of the information pick-up by experts and non-experts remained essentially unchanged when the visual display was degraded from normal video to point-light. Thus both experts and novices can utilise pure kinematic information at fairly coarse resolution (26 spatial co-ordinates representing positions of key joint centres, shuttle and racket, $24 \mathrm{fps}$ ) in action prediction. Our overall aim was to analyse the neural basis of expertise in sports anticipatory skill; therefore, an important criterion for any putative neural substrate for anticipatory skill is that it should respond similarly to both point-light and normal video display types. 
Much is known about the cortical networks that mediate the perception of, and responses to, others' actions, but these systems have been relatively little studied in the context of sporting performance. Perceptual skill in sport has some very specific task requirements that extend beyond mere action observation; for example, action prediction. The observation of body movements is known to activate the superior temporal sulcus (STS), plus a network of brain areas including the posterior inferior frontal gyrus (IFG), the rostral inferior parietal lobule (IPL), and the intraparietal sulcus (IPS). This is proposed as the core network of the mirror-neuron system (MNS) $[9,10,11,16,18,19]$. Wright and Jackson [22] used temporal occlusion to study cortical fMRI activation in predicting the direction of a tennis serve. Relative to a passive, actionobservation control condition, action prediction activated the MNS. Wright, Bishop, Jackson and Abernethy [23] found stronger activations for early-occluded than for late-occluded sequences of a badminton shot, particularly in premotor areas of the MNS and in medial frontal cortex (MFC). Moreover, experts showed greater activation than novices in the frontal MNS and MFC, with early-occluded sequences. Similar associations between the MNS and expertise were found in a study of elite basketball players [6].

On the basis of the studies reviewed above, we put forward four hypotheses concerning wholebrain fMRI responses to a sports anticipation task relative to control stimuli: (1) if anticipation depends on kinematic information, then a similar set of brain areas including, but not limited to, MNS will be activated by both point-light and normal video display types; (2) stronger activation in experts than in novices is predicted in these task-sensitive areas, for both display types; (3) expertise effects are predicted for fMRI responses to early- versus late-occluded sequences, and (4) there will be differences in fMRI responses due to display type in cortical visual areas, but these differences will be reduced or absent in other task-sensitive areas.

\section{Method}

\section{Stimuli}

We filmed video sequences of skilled badminton players executing an overhead clear shot to each of four court quadrants from the opponent's mid-court viewpoint, and control video clips of between-play (no-shot) movements. Point-light videos were produced by filming the same players in black tight-fitting clothing onto which strips of reflective tape were attached, representing 19 key joints and body parts (i.e., toes, ankles, knees, hips, shoulders, elbows, wrists, forehead, plus the sides, neck and head of the racket). During filming, two 500 Watt floodlights were directed to the actors, and the camera (Panasonic NV GS400) shutter speed and aperture were adjusted to maximise contrast between the reflective strips and clothing. The video sequences were postproduced using video editing software (Pinnacle Studio Pro, v. 11.0) to enhance brightness and contrast. Video clips were edited to create two levels of temporal occlusion for each video format; $160 \mathrm{~ms}$ before and $80 \mathrm{~ms}$ after racket-shuttle contact.

\section{Procedure}

The research was approved by the University Ethics Committee in accordance with the Declaration of Helsinki. All participants gave written informed consent and underwent pre-scan screening. Stimuli were back-projected at the rear of the scanner and viewed via an overhead mirror. Participants pressed a button to indicate the court quadrant (near left, far left, near right or far right) in which the shuttle would land. Each experiment was run twice: once using normal video sequences and once using point-light sequences (order counterbalanced). The block design 
of each run was identical. Participants practiced the mapping of response buttons to court positions in advance of the scanner session, and reminders were given by showing an annotated picture of the court after every 8 blocks. For control trials, participants pressed a button to indicate that no shot was played. We reminded participants to respond promptly on every trial but emphasised accuracy over speed.

\section{Participants}

Participants were recruited on a continuous basis through advertisement and professional contacts and all completed a questionnaire of their playing history and demographic details. Recruitment for the present study continued until a quota sample of 24 male participants was achieved, consisting of 8 experts (national/international level competitors), 8 intermediates (university/local league players) and 8 novices (limited experience). Participants were aged 18-28 years ( $M 22.9$ yrs, $S D 2.9$ yrs).

\section{fMRI Data Acquisition}

We acquired functional and structural images on a Trio 3T MRI scanner (Siemens, Erlangen, Germany) via an eight-channel array head coil. For each functional run, a standard, whole brain, echo planar gradient-echo imaging sequence was used to acquire 41 transverse slices $(3 \mathrm{~mm}$ thickness, TR $3000 \mathrm{~ms}$, TE $31 \mathrm{~ms}$, flip angle $=90^{\circ}$ ). Whole brain anatomical data were collected using a 176 slice, $1 \mathrm{~mm}^{3}$ voxel size, MP-RAGE T1-weighted sequence.

\section{Design}

The block design comprised 4 experimental conditions (block types): late occluded sequences (terminating $80 \mathrm{~ms}$ after the racket-shuttle contact), early occluded sequences (terminating 160 ms before), mctrl: no-shot motion control sequences (clips of the badminton player between play, moving legs and arms and swinging the racket), and sctrl: no-shot freeze-frames from the motion control. Each block consisted of five 1.76 s video clips of badminton play, each followed by a 2.4 s blank grey screen. There were eight interleaved blocks of each type within an experiment. We used the same block design for both display types.

\section{Data analysis}

We analysed fMRI data using SPM8 (http://www.fil.ion.ac.uk/spm). Functional images were spatially realigned to the first image in the series then co-registered with the T1 image. Images were normalised to the Montreal Neurological Institute (MNI) template then smoothed using a Gaussian kernel of $6 \mathrm{~mm}$ full-width half-maximum. The design matrix convolved the experimental design with a hemodynamic response function. The model was estimated using proportional scaling over the session to remove global effects, and with a high pass filter of $128 \mathrm{~s}$. For each individual, we computed t-contrasts for late-mctrl and early-mctrl. Thereafter we conducted whole-brain, second-level, random-effects analyses on t-contrasts from the individual data. We allocated anatomical labels and Brodmann areas to functional data using WFU PickAtlas [15]. 
Results

\title{
Behavioural data
}

We tested the effects of display type, occlusion and expertise on accuracy. The within-participant variables were display type (normal, point-light) and occlusion (early, late). Competitive level (novice, intermediate, expert) was entered as a between-participants factor in a 3-way ANOVA. There was a significant main effect of expertise, $F(2,21)=7.9, p<0.005$; partial $\eta^{2}=0.43$. Posthoc contrasts (Tukey HSD) showed that experts, $p<0.01$ and intermediates, $p<0.05$ were significantly more accurate than novices, but the difference between experts and intermediates, did not reach significance. Late occlusion performance was more accurate than for early occlusion, $F(1,21)=98.1, p<0.0001$; partial $\eta^{2}=0.82$. These results are consistent with previous literature, but no significant effects of display type or interactions were found $[1,4,5]$.

\section{fMRI responses to anticipation task with normal video and point-light stimuli}

To test Hypothesis 1 we analysed the activations during prediction of stroke direction, relative to observation of between-play movements ( $m c t r l$ ), in a three-way mixed ANOVA. The factors were expertise (novice, intermediate, expert), display type (normal video, point-light) and occlusion (early, late). Our first objective was to identify significant brain activity related to the direction prediction task across all participants (Table 1).

Table 1 near here

\begin{abstract}
Although there were significant effects of occlusion, active clusters for early and late occlusion overlapped. Clusters that responded significantly for both early-mctrl and late-mctrl contrasts in the normal video condition are shown in Figure 1 (upper row, green). We found a very similar pattern of activation for the point-light replication (Figure 1, lower, green). A horizontal section at $\mathrm{Z}=50$ or $\mathrm{z}=40$ (Figure 1, upper and lower rows) reveals four discrete regions of activation (a-d) mirrored in each hemisphere and located in, respectively, MFC, premotor cortex, IPL, and SPL. At $\mathrm{z}=30$ we located cluster (e) in IFG; at $\mathrm{z}=20$, (f) in DLPFC; at $\mathrm{z}=10$, (g) in STS; and at $\mathrm{z}=$ 0 , clusters $(\mathrm{h})$ in anterior insula and frontal operculum. Areas that responded more strongly to the control condition (action observation) than the experimental condition (action prediction) are shown in yellow. These include posterior cingulate (i) and medial occipital cortex (j). Statistical results and MNI coordinates of peak activations (Table 1) of these clusters showed that anticipation of the aim of a badminton shot (relative to observation of between-play movements of the same player) activates a consistent set of brain areas, mainly bilateral, and including the core MNS. Overall, Hypothesis 1 was confirmed: point-light and normal video results were similar and both activated the MNS.
\end{abstract}

Figure 1 near here

To test Hypothesis 2, t-contrasts were computed for expert and intermediate groups versus novices: results are shown in Figure 1 and Table 2. For both full video and point-light display types, voxels showing positive effects of expertise (Figure 1 blue) corresponded with the areas responding to the task contrasts, early-mctrl and late-mctrl (green), confirming our second hypothesis. However the peak of the expert-novice difference did not always coincide with the peak of the task-related activation: for SPL it was more posterior, and for IPL and MT/MST it was more ventral. Conversely, positive effects of expertise were absent from areas (yellow) that 
responded more strongly to the control condition (action observation) than to the task. These include areas of medial occipital cortex $(\mathrm{h})$ and anterior and posterior cingulate cortex. Some voxels in these areas respond more strongly in novices than experts (magenta). The statistical results and MNI coordinates of cluster peaks for expert-novice differences are shown in Table 2 both for normal video and for point-light stimuli.

Table 2 near here

\section{Interactions between expertise and level of occlusion}

ANOVA also indicated significant interactions between expertise and the occlusion variable (early-mctrl and late-mctrl). Figure 2 shows the positive interaction of expertise with early and late occlusion across both display types. The significant voxels lie within task-sensitive areas but are predominantly anterior for early occlusion and posterior for late occlusion. For example, IFG, MFG and especially DLPFC show more extensive expertise-related responses to early occlusion and SPL and MT/MST+ to late occlusion. Thus Hypothesis 3 is supported, and there is some evidence for anterior-posterior specialization in relation to analysis of early and late cues, respectively.

Figure 2 near here

\section{Effects of display type (normal video versus point-light).}

Hypothesis 4 supposed that differences due to display type would predominate in visual cortical areas, rather than higher-level task-sensitive areas. This was confirmed, as the only active voxels (at $p<0.005$ uncorrected) were in medial occipital cortex $(\mathrm{BA} 18,19)$ and in BA 30. There were no significant voxels responding, at $p<0.005$ uncorrected, to the interaction of expertise with display type. Overall, expert and novice brains did not differ in their responses to normal and point-light stimuli.

\section{Discussion}

There are three main conclusions from the present study. First, those areas that respond similarly to normal and point-light video in the anticipation task can be considered specific to sports-related anticipation. This includes substantial parts of MNS and visual attention systems. Differences between normal and point-light video were found only in areas that are not task-specific in their pattern of activation, such as visual cortex. This suggests that in the conditions of the present study, only a basic kinematic representation is passed forward to the MNS.

Second, whole-brain analyses provide a more comprehensive delineation of brain systems concerned with anticipation skills than our previous approach based on a priori regions of interest [23]. We identify new regions showing expertise effects within SPL, MT/MST, STS, DLPFC and anterior insula. For some of these areas, the peak of the expert-novice difference is spatially distinct from the peak of the task-related activation. Areas responding more strongly to the control action observation condition than the anticipation task included visual areas $($ BA18, 19) and elements of a proposed default mode network [12] (retrosplenial, temporoparietal and anterior cingulate cortex) and within some of these areas there were clusters activated more in novices than experts. This is arguably a deactivation effect [20], with stronger suppression of taskunrelated stimuli in experts. 
Thirdly, our results suggest specialization within the network of task-activated areas. Expertise effects in our data show an anterior (early) - posterior (late) bias for occluded sequences. For example SPL may be utilised more in expert brains for orientation towards later visual cues (including the start of shuttle flight) whereas premotor cortex and MFC may be utilised more in analysis of early body kinematics (Figure 2). The involvement of DLPFC in early occlusion suggests greater cognitive control by experts in the more difficult condition.

Comparison of current findings with published studies indicates the involvement of both the MNS and visual attention systems. Observed goal-directed actions are thought to be processed without conscious effort, through activation of the MNS $[9,10,11,16,18,19]$ and this constitutes the first of our two interlocking systems, comprising IPL, premotor cortex, IFG and pre-SMA. This system was active both in experts and novices observing our stimuli. Sport expertise effects would be expected within this system if relevant connections between MNS neurons in IPL and IFG are strengthened through associative learning [14]. MT/MST was consistently activated by task stimuli in both normal video and point-light versions, and the active cluster often extended beyond MT/MST itself into STS, suggesting contiguous areas responding to biological motion stimuli [21], consistent with a role providing biological motion input to the MNS via parietal cortex [9]. Expert-novice differences were found on the ventral edge of the MT/MST complex (Figure 1). Conversely, in our previous regions of interest study based on MT/MST functional localiser [23], no expert-novice differences were found; thus the expertise effects may be confined to a subdivision or accessory of this complex.

Other areas showing task-related activity in our results are not usually considered to be part of the MNS. In "ocular baseball" anticipation tasks with abstract stimuli [13] SEF and FEF activations reflected both decision-making and oculomotor processes, whereas SPL (BA7) and frontal operculum/anterior insula (BA47/13) responded to a go/nogo decision regardless of whether an eye movement was executed [13]. We found anticipation-related activations and expert-novice differences in all of these areas except FEF, but they appear to form part of a visual attention network rather than a MNS network [8]. The deployment of spatial attention is intrinsic to many visuospatial and oculomotor tasks, including anticipation in sport.

Attention and MNS systems are closely related: IFG responses to action observation are suppressed by a competing attention load [7]. It is unlikely that expert-novice differences in our study are related to differences in attention systems themselves [17] but rather to the way these are used in the task of analysing body kinematics to predict a shot. We also suggest a strong link between action observation and attention, in that the intentional movements of other human beings can be compelling environmental cues for the direction of attention.

\section{Conclusions}

In a sports anticipation task, experts show stronger fMRI activations than novices in brain areas that are associated with visual attention and the analysis of body kinematics. Conversely, novices show stronger responses in occipital cortex suggesting a greater allocation of resources to lowlevel visual processing. Results using point-light stimuli replicate the main results from normal video sequences, indicating the sufficiency of kinematic information for the working of these cortical mechanisms.

Acknowledgements 
The work described in this paper was supported by a grant from the Research Grants Council of the Hong Kong Special Administrative Region, China, Project HKU 7400/05H.

\section{References}

[1] Abernethy, B. (1990). Expertise, visual search and information pick-up in squash. Perception, 19, 63-77.

[2] Abernethy, B., Gill, D., Parks, S.L. \& Packer, S.T. (2001). Expertise and the perception of kinematic and situational probability information. Perception, 30, 233-252.

[3] Abernethy, B. \& Russell, D.G. (1987). The relationship between expertise and visual search strategy in a racket sport. Human Movement Science, 6, 283-319.

[4] Abernethy, B., \& Zawi, K. (2007). Pickup of essential kinematics underpins expert perception of movement patterns. Journal of Motor Behavior, 39 (5), 353-367.

[5] Abernethy, B., Zawi, K. \& Jackson, R. C., (2008). Expertise and attunement to kinematic constraints. Perception, vol. 37, 931 - 948.

[6] Aglioti, S.M., Cesari, P., Romani, M. \& Urgesi, C. (2008). Action anticipation and motor resonance in elite basketball players. Nature Neuroscience, 11, 1109-1116.

[7] Chong, T.T.-J., Williams, M.A., Cunnington, R., Mattingley, J.B. (2008) Selective attention modulates inferior frontal gyrus activity during action observation. NeuroImage, 40 (1), 298-307.

[8] Corbetta, M. \& Shulman, G.L. (2002). Control of goal-directed and stimulus-driven attention in the brain. Nature Reviews Neuroscience, 3, 201-215.

[9] Decety, J. \& Grèzes, J. (1999). Neural mechanisms subserving the perception of human actions. Trends in Cognitive Science, 3,172-178.

[10] Filimon, F., Nelson, J.D., Hagler, D.J. \& Sereno, M.I. (2007). Human cortical representations for reaching: mirror neurons for execution, observation and imagery. Neuroimage, $37,1315-1328$.

[11] Grèzes, J. \& Decety, J. (2001). Functional anatomy of execution, mental simulation, observation and verb generation of actions: a meta-analysis. Human Brain Mapping, 12, 1-19. [12] Gusnard, D.A. \& Raichle, M.E. (2001). Searching for a baseline: functional imaging and the resting human brain. Nature Reviews Neuroscience, 2, 685-694.

[13] Heinen, S.J., Rowland, J., Lee, B.-T., and Wade, A.R. (2006). An oculomotor decision process revealed by functional magnetic resonance imaging. Journal of Neuroscience, 26, 1351513522

[14] Heyes, C. (2010). Where do mirror neurons come from? Neuroscience and Biobehavioral Reviews, 34, 575-583.

[15] Maldjian, J.A., Laurientini, P.J., Burdette, J.B. \& Kraft, R.A. (2003). An automated method for neuroanatomic and cytoarchitectonic atlas-based interrogation of fMRI data sets. NeuroImage, $19,1233-1239$.

[16] Morin, O, \& Grèzes J. (2008). What is "mirror" in the premotor cortex? A review. Neurophysiologie Clinique/Clinical Neurophysiology. 38, 189-195

[17] Overney, L.S., Blanke, O. \& Herzog, M.H. (2008). Enhanced temporal but not attentional processing in expert tennis players. PLoS ONE, 3 (6), e2380.

[18] Rizzolatti, G. \& Craighero, L (2004). The mirror-neuron system. Annual Review of Neuroscience, 27, 169-192.

[19] Rizzolatti, G., Fogassi, L., \& Gallese, V. (2001). Neurophysiological mechanisms underlying the understanding and imitation of action. Nature Reviews Neuroscience, 2, 661-670.

[20] Shulman, G.L., Astafiev, S.V., McAvoy, M.P., d'Avossa, G., \& Corbetta, M. (2007). Right TPJ deactivation during visual search: functional significance and support for a filter hypothesis. Cerebral Cortex, 17, 2625-2633. 
[21] Thompson, J. C., Clarke, M., Stewart, T., \& Puce, A. (2005). Configural processing of biological motion in human superior temporal sulcus. Journal of Neuroscience, 25, 9059-9066. [22] Wright, M. J. \& Jackson, R. C. (2007). Brain regions concerned with perceptual skills in t ennis: an fMRI study. International Journal of Psychophysiology, vol. 63, 214-220

[23] Wright, M.J., Bishop, D.T., Jackson, R.C., Abernethy, B. (2010). Functional MRI reveals expert-novice differences during sport-related anticipation. Neuroreport, 21, 94-98.

Figure 1: The top two rows show responses to normal video, and the bottom two to point-light video. Functional data are superimposed on horizontal sections of the average T1 image, shown at $10 \mathrm{~mm}$ intervals from $\mathrm{z}=60$ to $\mathrm{z}=-10$. Green: clusters responding significantly more to badminton shots than to between-play control clips; and Yellow: clusters responding more to controls than to shots (both $>10$ voxels $p<0.001$ FDR corrected, all participants). Blue: clusters responding more strongly in experts than novices. Magenta: clusters responding more strongly in novices than experts (both $>10$ voxels, $p<0.001$ uncorrected). a: MFC, b: premotor cortex, c: IPL, d: SPL, e: IFG, f: DLPFC, g: STS, h: anterior insula and frontal operculum, i: posterior cingulate, $\mathrm{j}$ : medial occipital cortex.

Figure 2: Voxels responding more strongly in experts than novices for early-occluded (cyan) and late-occluded (magenta) badminton sequences (both $>10$ voxels, $p<0.005$ uncorrected) for both display types combined. Letter labels and anatomical sections are as for Figure 1. 


\section{Figure}

Click here to download high resolution image

\section{normal video}
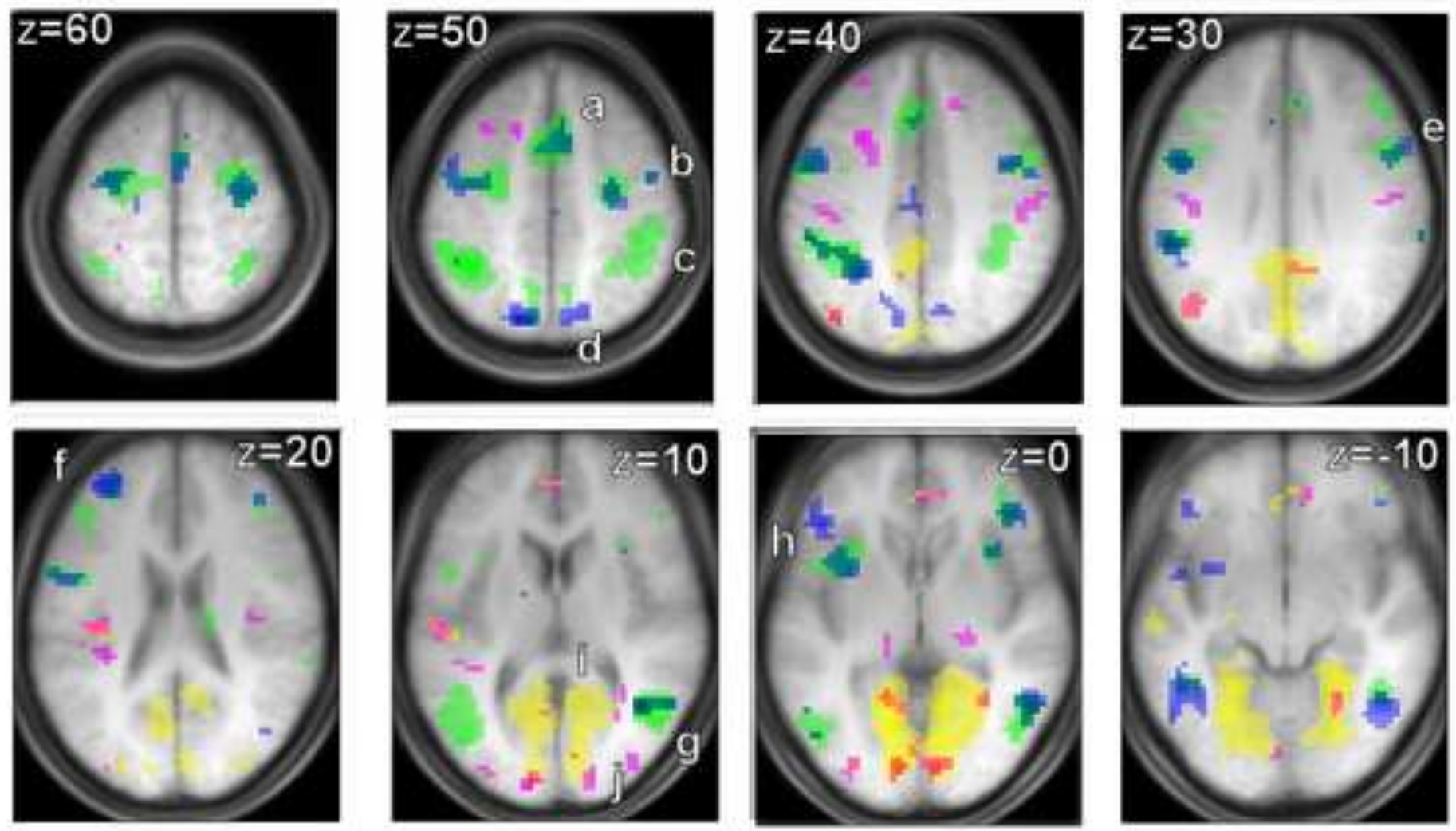

point-light video
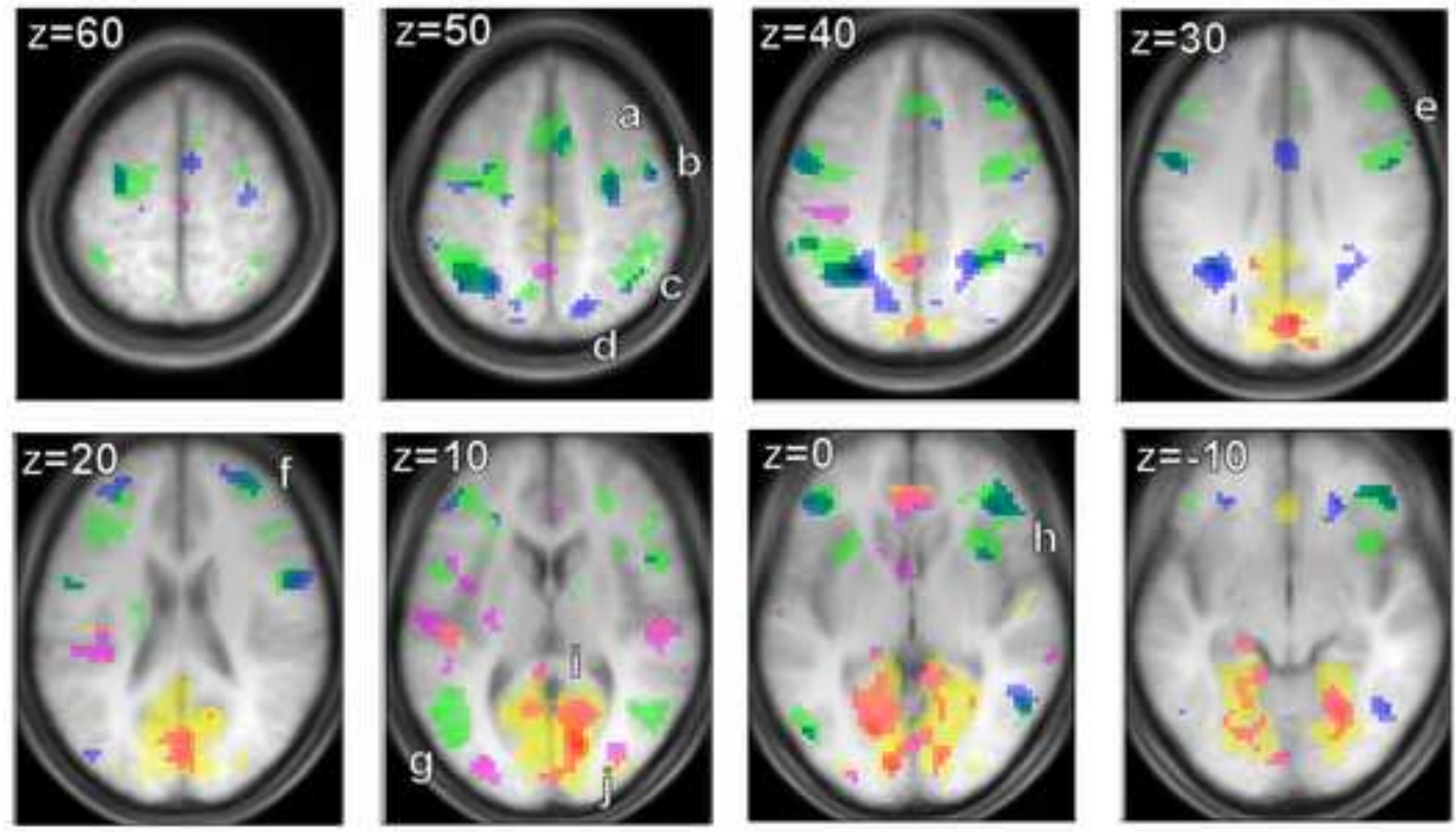

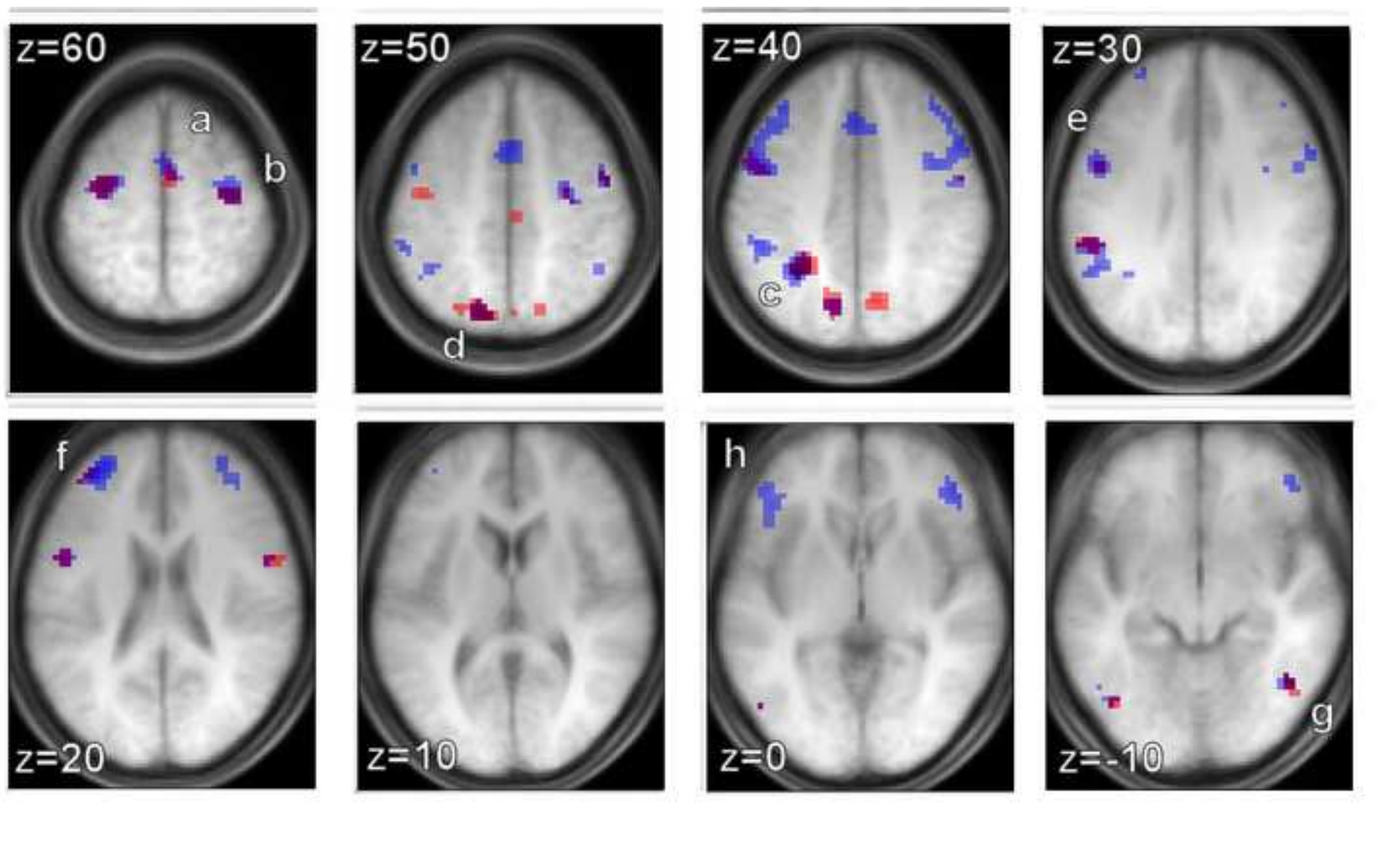

\section{.}

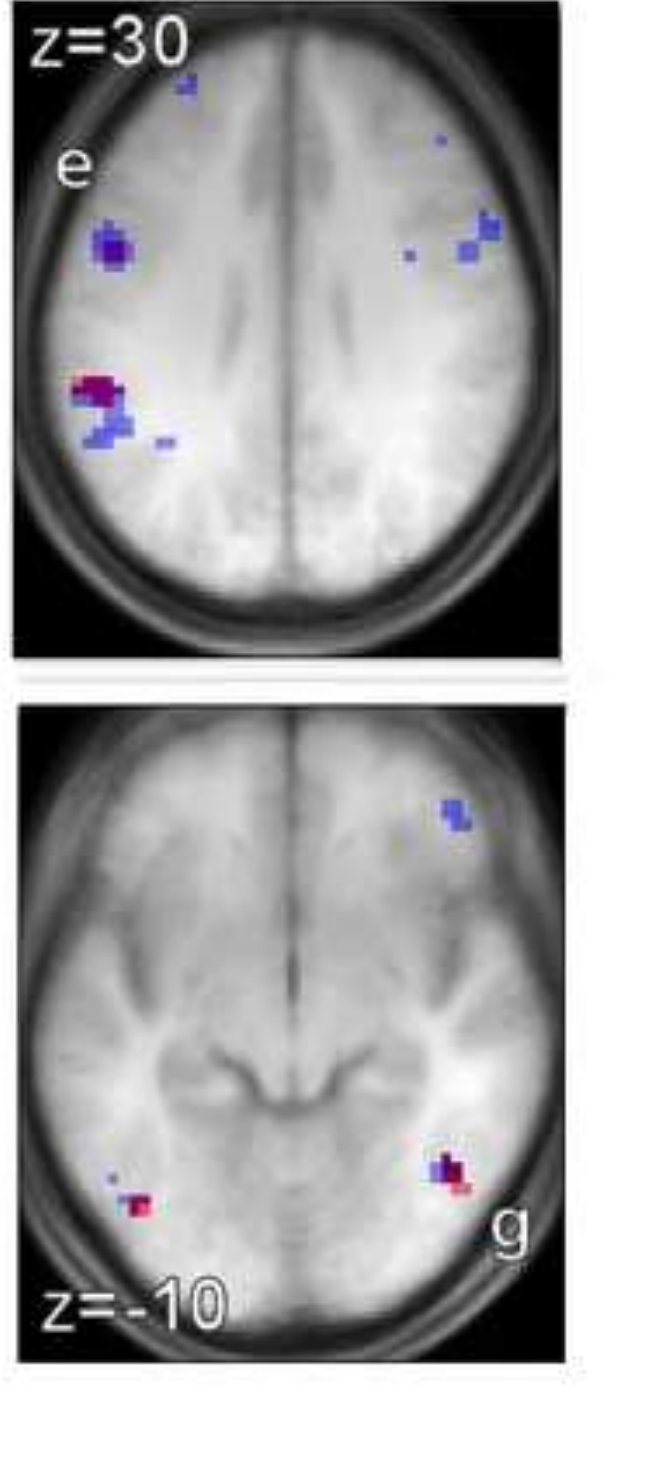




\begin{tabular}{|c|c|c|c|c|c|c|c|c|c|c|c|c|}
\hline \multirow[b]{2}{*}{ Area } & \multirow[b]{2}{*}{$\mathrm{BA}$} & \multirow[b]{2}{*}{ Fig.1 } & \multicolumn{5}{|c|}{ Normal video } & \multicolumn{5}{|c|}{ Point-light } \\
\hline & & & cluster & $\mathrm{x}$ & $\mathrm{y}$ & $\mathrm{Z}$ & $Z$ & cluster & $\mathrm{x}$ & $\mathrm{y}$ & $\mathrm{Z}$ & $Z$ \\
\hline L IPL & 40 & $\mathrm{c}$ & 561 & -39 & -48 & 51 & Inf & 504 & -39 & -48 & 51 & Inf \\
\hline R IPL & 40 & & 430 & 33 & -48 & 57 & 7.3 & 385 & 51 & -42 & 45 & 7.0 \\
\hline \multirow[t]{2}{*}{ L premotor } & 6,9 & $\mathrm{~b}$ & 753 & -27 & -9 & 57 & 7.6 & 978 & -48 & 6 & 12 & Inf \\
\hline & 6 & & & -51 & 6 & 15 & 7.2 & & -27 & -9 & 57 & 6.4 \\
\hline \multirow[t]{2}{*}{$\mathrm{R}$ premotor } & 6,9 & & 436 & 51 & 9 & 36 & 7.0 & 186 & 51 & 6 & 21 & 6.3 \\
\hline & 6 & & 187 & 33 & -3 & 57 & 7.2 & 562 & 30 & -9 & 51 & 7.5 \\
\hline L \& R MFC & 8 & $\mathrm{a}$ & 388 & 0 & 9 & 51 & Inf & & 0 & 30 & 39 & 7.3 \\
\hline L STS & 37,39 & $\mathrm{f}$ & 288 & -51 & -69 & 3 & 7.7 & 224 & -54 & -63 & 6 & 7.7 \\
\hline R STS & 37 & $\mathrm{f}$ & 292 & 48 & -63 & 9 & 7.7 & 105 & 54 & -63 & 9 & 6.7 \\
\hline L IFG & 47 & & & & & & & 182 & -42 & 45 & 6 & 6.2 \\
\hline R IFG & 47 & $\mathrm{e}$ & 24 & 45 & 51 & -4 & 6.3 & 286 & 45 & 48 & -9 & 7.0 \\
\hline DLPFC & 10,46 & $\mathrm{~g}$ & 14 & 45 & 39 & -3 & 6.7 & 332 & -27 & 45 & 18 & 6.8 \\
\hline L \& R SPL & 7 & $\mathrm{~d}$ & 152 & -6 & -63 & 57 & 5.9 & 49 & -6 & -60 & 54 & 6.8 \\
\hline $\mathrm{L}$ ant insula & 13,47 & & 17 & -30 & 21 & 3 & 6.6 & 35 & -33 & 15 & 3 & 6.4 \\
\hline $\mathrm{R}$ ant insula & 13,47 & $\mathrm{~h}$ & 40 & 33 & 21 & 0 & 6.0 & & 33 & 21 & 3 & 6.6 \\
\hline R caudate & & & 24 & 15 & -15 & 21 & 5.5 & 25 & 15 & 0 & 15 & 5.9 \\
\hline
\end{tabular}

Table 1b. MNI co-ordinates of significant clusters ( $>10$ voxels, $p<0.001$ FDR corrected) representing the negative response to early-mctrl and late-mctrl t-contrasts across all participants.

\begin{tabular}{|lc|rrrrr|rrrrr|}
\hline $\begin{array}{l}\text { L \& R medial } \\
\text { occipital }\end{array}$ & 18,23 & 2939 & -12 & -84 & -3 & Inf & 4114 & 12 & -63 & 18 & Inf \\
& & & -15 & -57 & 0 & Inf & & 12 & -75 & 9 & Inf \\
\hline L TPJ & 39 & 37 & -48 & -66 & 36 & 7.2 & & & & & \\
\hline $\begin{array}{l}\text { L MT/MST } \\
\text { R MT/MST }\end{array}$ & 19 & 62 & 36 & -84 & 15 & 6.5 & & & & & \\
\hline L MTG & 21 & 91 & -60 & -18 & -12 & 6.9 & 39 & -57 & -6 & -12 & 5.6 \\
\hline $\begin{array}{l}\text { L mid insula } \\
\text { R mid insula }\end{array}$ & 13 & 119 & -36 & -21 & 21 & 6.2 & 77 & -42 & -18 & 24 & 6.1 \\
\hline $\begin{array}{l}\text { L \& R } \\
\text { temporal }\end{array}$ & 13 & & & & & & 23 & 39 & -18 & 21 & 6.0 \\
\hline ant cingulate & 32,10 & 44 & 63 & -6 & -18 & & 24 & -45 & -24 & 12 & 6.2 \\
\hline
\end{tabular}


Table 2. Locations and statistics of voxel clusters responding to the positive effect of expertise $(A)$ and the negative effect of expertise $(B)$ at $p<0.001$ uncorrected.

\begin{tabular}{|c|c|c|c|c|c|c|c|c|}
\hline$A$ & & & Normal & leo $\operatorname{Exp}>N$ & & Point-lig & it Exp > Nov & \\
\hline Area & BA & Fig.1 & cluster & $\begin{array}{lll} & y & z\end{array}$ & $Z$ & cluster & $\begin{array}{lll}x & y & z \\
\end{array}$ & $Z$ \\
\hline L premotor & 9,44 & $\mathrm{~b}$ & 90 & $\begin{array}{lll}-54 & 3 & 36\end{array}$ & 5.6 & 53 & $\begin{array}{lll}-54 & 3 & 33\end{array}$ & 4.6 \\
\hline & 6 & & 64 & $\begin{array}{lll}-30 & -6 & 60\end{array}$ & 4.2 & 19 & $-33-12 \quad 66$ & 4.2 \\
\hline $\mathrm{R}$ premotor & 9 & & 33 & $\begin{array}{lll}51 & 9 & 33\end{array}$ & 4.3 & 25 & $\begin{array}{lll}57 & 6 & 21\end{array}$ & 4.5 \\
\hline & 6 & & 55 & $30-1254$ & 3.9 & 28 & $\begin{array}{lll}30 & -9 & 51 \\
\end{array}$ & 2.9 \\
\hline L IPL & 40 & $\mathrm{c}$ & 126 & $\begin{array}{lll}-57 & -33 & 33\end{array}$ & 4.7 & 327 & $-30-5136$ & 5.3 \\
\hline R IPL & 40 & & & & & 55 & $\begin{array}{lll}27 & -42 & 36 \\
\end{array}$ & 4.9 \\
\hline L SPL & 7 & $\mathrm{~d}$ & 61 & $-15-7251$ & 5.2 & & & \\
\hline R SPL & 7 & & 35 & $\begin{array}{lll}12 & -72 \quad 45 \\
\end{array}$ & 4.0 & 23 & $15-6648$ & 4.1 \\
\hline L DLPFC & 10,46 & $\mathrm{G}$ & 41 & $\begin{array}{lll}-33 & 51 & 21\end{array}$ & 5.2 & 29 & $\begin{array}{lll}-48 & 42 & 0\end{array}$ & 4.0 \\
\hline R DLPFC & 10 & & & & & 10 & $33 \quad 54 \quad 24$ & 3.7 \\
\hline L IFG & 47 & & 38 & $\begin{array}{lll}-48 & 33 & -3\end{array}$ & 4.5 & & & \\
\hline R IFG & 47 & e & 16 & $\begin{array}{lll}45 & 39 & -3\end{array}$ & 4.8 & 69 & $\begin{array}{lll}51 & 42 & -6\end{array}$ & 5.3 \\
\hline L MTG & 37,39 & $\mathrm{f}$ & 51 & $\begin{array}{lll}-51 & -57 & -6\end{array}$ & 4.3 & 10 & $\begin{array}{lll}-51 & -69 & -3\end{array}$ & 4.4 \\
\hline R MTG & 37,39 & & 86 & $\begin{array}{lll}42 & -57 & 9 \\
\end{array}$ & 4.6 & 31 & $\begin{array}{lll}48 & -60 & -9 \\
\end{array}$ & 3.9 \\
\hline $\mathrm{L} \& \mathrm{R} \mathrm{MFC}$ & 6 & $\mathrm{a}$ & 35 & $\begin{array}{lll}3 & -3 & 66\end{array}$ & 4.4 & & & \\
\hline & 8 & & 40 & $\begin{array}{lll}6 & 12 & 51\end{array}$ & 4.3 & & & \\
\hline & 9,32 & & & & & 12 & $\begin{array}{lll}9 & 15 & 45\end{array}$ & 3.4 \\
\hline $\mathrm{L}$ ant insula & 13 & & 40 & $\begin{array}{lll}-33 & 9 & -3\end{array}$ & 5.0 & & & \\
\hline $\mathrm{R}$ ant insula & 13 & & 10 & $\begin{array}{lll}33 & 18 & 3 \\
\end{array}$ & 4.1 & & & \\
\hline $\mathrm{R}$ cingulate & 31 & & 18 & $\begin{array}{lll}0 & -21 & 42 \\
\end{array}$ & 4.1 & & & \\
\hline $\mathrm{R}$ cingulate & 24 & & & & & 19 & $\begin{array}{lll}3 & 3 & 33 \\
\end{array}$ & 3.9 \\
\hline$B$ & & & Normal & leo Nov > E & & Point-lig & it Nov $>$ Exp & \\
\hline Area & $\mathrm{BA}$ & Fig.1 & cluster & $\begin{array}{lll}x & y & z \\
\end{array}$ & $Z$ & cluster & $\begin{array}{lll}x & y \quad z \\
\end{array}$ & $Z$ \\
\hline Occipital L & 18 & & 30 & $-12-9612$ & 4.9 & 118 & $\begin{array}{ccc}-18 & -69 & 0\end{array}$ & 4.7 \\
\hline & 19 & & 12 & $-27-8515$ & 3.6 & 46 & $\begin{array}{lll}-30 & -84 & 6\end{array}$ & 4.6 \\
\hline Occipital R & 17,18 & $\mathrm{j}$ & 35 & $\begin{array}{lll}9 & -87 & 3\end{array}$ & 4.5 & 34 & $\begin{array}{lll}3 & -72 & 24\end{array}$ & 3.4 \\
\hline & 19 & & & & & 14 & $33-7812$ & 3.7 \\
\hline R IPL & 39 & & 27 & $42-7239$ & 4.5 & & & \\
\hline L temporal & 41 & & & & & 108 & $-45-24 \quad 12$ & 4.4 \\
\hline & 42 & & 15 & $\begin{array}{lll}-57 & -33 & 15\end{array}$ & 3.5 & & & \\
\hline R temporal & 41 & & & & & 23 & $\begin{array}{lll}51 & -21 & 12\end{array}$ & 4.2 \\
\hline & 20 & & 22 & $54-12-24$ & 4.1 & & & \\
\hline Ant cingulate & 32 & & & & & 27 & $\begin{array}{lll}-3 & 42 & 3\end{array}$ & 4.5 \\
\hline Post cingulate & 31 & & & & & 19 & $\begin{array}{lll}0 & -48 & 36\end{array}$ & 4.0 \\
\hline L caudate & & & & & & 16 & $\begin{array}{lll}-3 & 9 & -3 \\
\end{array}$ & 4.1 \\
\hline Mid insula & 13 & & 13 & $39-15 \quad 21$ & 3.0 & & & \\
\hline Pulvinar & & & & & & 16 & $-18-30 \quad-3$ & 3.9 \\
\hline
\end{tabular}

